\title{
Quercetin Enhanced Paclitaxel Therapeutic Effects Towards PC-3 Prostate Cancer Through ER Stress Induction and ROS Production
}

This article was published in the following Dove Press journal: OncoTargets and Therapy

\author{
Xiangyu Zhang $\mathbb{D}^{1, *}$ \\ Jingwen Huang ${ }^{2, *}$ \\ Chao $\mathrm{Yu}^{3}$ \\ Longquan Xiang' \\ Liang Li ${ }^{\prime}$ \\ Dongmei Shi (iD) \\ Fanzhong Lin'
}

'Department of Pathology, Jining First People's Hospital, Jining Medical University, Jining 272000, People's Republic of China; ${ }^{2}$ Anhui Province Key Laboratory of Translational Cancer Research Affiliated to Bengbu Medical University, Bengbu 233004, People's Republic of China; ${ }^{3}$ Department of Clinical Medicine, Jining Medical University, Jining 272000, People's Republic of China; ${ }^{4}$ Department of Dermatology, Jining First People's Hospital, Jining Medical University, Jining 272000, People's Republic of China

*These authors contributed equally to this work

Correspondence: Dongmei Shi Department of Dermatology, Jining First People's Hospital, Jining Medical University, No. 6 Jiankang Road, Jining 272000, People's Republic of China $\mathrm{Tel} / \mathrm{Fax}+86537605 \mathrm{I} 547$

Email shidongmei28@163.com

Fanzhong Lin

Department of Pathology, Jining First People's Hospital, Jining Medical University, No. 6 Jiankang Road, Jining 272000, People's Republic of China Tel/Fax +865376051547

Email linfanzhongI@I26.com
Introduction: Prostate cancer is one of the most common cancers threatening public health worldwide. Although chemotherapy plays an important role in treating prostate cancer, it leads to many adverse effects and is prone to drug resistance. Quercetin, a natural product, is used in traditional Chinese medicine because of its strong antitumor activity and few side effects.

Methods: In this study, we combined quercetin and paclitaxel to kill prostate cancer cells in vivo and in vitro, and we investigated the relevant mechanism of this combination treatment. After the cancer cells were treated with quercetin or/and paclitaxel, cell growth inhibition, apoptosis, the cell cycle, reactive oxygen species (ROS) generation, and several endoplasmic reticulum (ER) stress signaling pathway related gene expressions were evaluated.

Results: The combined treatment with quercetin and paclitaxel significantly inhibited cell proliferation, increased apoptosis, arrested the cell cycle at the G2/M phase, inhibited cell migration, dramatically induced ER stress to occur, and increased ROS generation. In a PC-3 cancer-bearing murine model, this combination treatment exerted the most beneficial therapeutic effects, and quercetin increased the cancer cell-killing effects of paclitaxel, with nearly no side effects compared with the single paclitaxel treatment group.

Conclusion: Combination treatment possessed enhanced anti-cancer effects, and these results will provide a basis for treating prostate cancer using a combination of quercetin and paclitaxel.

Keywords: quercetin, paclitaxel, combination treatment, endoplasmic reticulum stress, reactive oxygen species

\section{Introduction}

Prostate cancer, which has a high incidence and mortality rate worldwide, has shown an increasing incidence in China. ${ }^{1,2}$ When the disease is diagnosed at the early stages, there is a high likelihood of a successful cure, especially using surgical resection or castration therapy. However, when the disease has progressed to an advanced stage, it is often fatal and leads to high mortality in men; thus, chemotherapy plays an important role in the advanced stages of prostate cancer. ${ }^{3}$ Despite significant primary chemosensitivity, prostate cancer can relapse subsequently, at which point chemotherapy becomes less effective because of chemo-resistance. Reversing this phenomenon may improve the outcomes of prostate cancer. ${ }^{4}$

Antimitotics, which target cellular tubulin, are among the most useful chemotherapeutic agents. Some tubulin-stabilizing agents that target the taxane-binding site are already available for clinical use, including paclitaxel, docetaxel, and epothilones. ${ }^{5}$ 
Paclitaxel (PTX) is a widely used chemotherapeutic agent for treating many types of cancers, including prostate, breast, ovarian, and lung cancers. PTX induces apoptosis by disrupting the dynamic equilibrium between soluble tubulin dimers and polymerized tubulin, inhibiting the cell transition from metaphase to anaphase. ${ }^{6}$ In addition, some clinical trials have verified that PTX has increased the survival rates of patients with prostate cancer. However, PTX exerts many adverse effects and can induce acquired drug resistance after treatment, thus inhibiting its clinical anticancer use. ${ }^{7}$

Some chemical synthetic agents that can reverse drug resistance have been tested in clinical trials, but many have failed due to their adverse effects. ${ }^{8,9}$ However, some natural products can avoid this problem. Quercetin (Que), a flavonoid that is a permanent component in human diets as well as an agent in traditional Chinese medicine, has been widely used to treat cancer for a longtime. ${ }^{10,11}$ However, the exact antitumor mechanism of Que is unclear. Several studies have showed that Que increased reactive oxygen species (ROS) production in hepatocellular cancer and prevented lipid oxidation in the cell membrane, whereas other studies have indicated that Que could increase apoptotic rates in cancer cells. ${ }^{12,13}$ Meanwhile, it was reported that Que downregulated heterogeneous nuclear ribonucleoprotein A1 (hnRNPA1) expression and sensitized enzalutamide to kill prostate cancer cells; thus, Que and enzalutamide worked synergistically in this treatment. ${ }^{14}$

In this study, we combined Que and PTX to treat prostate cancer, and we evaluated the in vitro and in vivo antitumor effects. In the in vitro study, we analyzed cancer cell proliferation, apoptosis, cell cycle arrest, and ROS production after the cancer cells were treated with both Que and PTX. We also studied endoplasmic reticulum (ER) stress and migration ability in cells. In the in vivo study, we analyzed the combination treatment effects in the PC-3 cancer-bearing mice, and we evaluated possible anti-cancer mechanism via immunohistochemistry staining of some relevant proteins. These studies may help to elucidate the antitumor mechanism of combined Que and PTX use. This combination treatment may effectively decrease the PTX dose in prostate cancer clinical therapy.

\section{Materials and Methods}

\section{Materials}

Rabbit anti-human GRP78 polyclonal antibody, mouse antihuman CHOP monoclonal antibody, mouse anti-human
hnRNPA1 monoclonal antibody, mouse anti-human cleaved caspase-3 monoclonal antibody, and anti-GAPDH monoclonal antibody were purchased from Abcam. Que and PTX were purchased from Dalian Meilun Biotechnology Co., Ltd. TRIzol $^{\circledR}$ reagent was purchased from Invitrogen (Gibco, Shanghai, China), and 3-(4,5-dimethylthiazol-2-yl)-2,5-diphenyltetrazolium bromide (MTT) was purchased from SigmaAldrich (Shanghai, China).

Human prostate cancer (PC-3) cell lines were obtained from the Cell Bank of the Chinese Academy of Sciences (Shanghai, China) and were grown in Dulbecco's modified Eagle's medium (DMEM) (Paisley, UK) containing 10\% fetal bovine serum (FBS) at $37^{\circ} \mathrm{C}$ in a humidified environment containing $5 \% \mathrm{CO}_{2}$.

Nude male BALB/c mice (4-6 weeks old, weighing 14-21 g) were purchased from Jining Medical University (Jining, China). All the animal procedures were performed in accordance with the National Institute of Health guidelines and were approved by the Jining First People's Hospital of Jining Medical University (Medical ethics committee of the Jining First People's Hospital of Jining Medical University).

\section{Cell Viability Analysis}

The cytotoxicity of Que and Que combined with PTX on PC-3 cells was evaluated using an MTT assay. The PC-3 cells were seeded onto 96-well plates at a concentration of $5 \times 10^{3}$ cells/well and incubated for $24 \mathrm{~h}$. The cells were incubated for $24 \mathrm{~h}$ using different concentrations of Que $(7.5,15,30,60,120 \mu \mathrm{M})$, PTX $(6.25,12.5,25,50,100$ $\mathrm{nM})$, and combined quercetin and paclitaxel $(7.5 \mu \mathrm{M}+6.25$ $\mathrm{nM}, 15 \mu \mathrm{M}+12.5 \mathrm{nM}, 30 \mu \mathrm{M}+25 \mathrm{nM}, 60 \mu \mathrm{M}+50 \mathrm{nM}, 120$ $\mu \mathrm{M}+100 \mathrm{nM})$. Next, an MTT-water solution (100 $\mu \mathrm{L}, 5 \mathrm{mg} /$ $\mathrm{mL}$ ) was added to each well and incubated for $4 \mathrm{~h}$. After incubation, the culture medium was completely replaced with $100 \mu \mathrm{L}$ of dimethylsulfoxide (DMSO) and incubated for $10 \mathrm{~min}$. The absorbance was measured at $490 \mathrm{~nm}$ using a microplate reader from Bio-Rad Laboratories (California, USA). We also tested the cytotoxicities of various concentrations of Que, PTX, and Que+PTX against the PC-3 cells using the same method. The Chou and Talalay method was used for the analysis of the Combination Index (CI) of the Que and PTX combination using CompuSyn software ((Combo Syn Inc., Paramus, NJ, USA).

\section{Intracellular ROS Content Assays}

Approximately $3 \times 10^{3}$ PC-3 cells per well were seeded onto 96-well plates. After approximately $24 \mathrm{~h}$, the cell density 
reached the required $70-80 \%$. Four groups were evaluated: control group, Que group ( $20 \mu \mathrm{M}$, the concentration of Que was $20 \mu \mathrm{M})$, paclitaxel group ( $5 \mathrm{nM}$, the concentration of PTX was $5 \mathrm{nM}$ ), and the combinational quercetin+paclitaxel group $(20 \mu \mathrm{M}+5 \mathrm{~nm}$, the concentration of quercetin was 20 $\mu \mathrm{M}$ and the concentration of paclitaxel was $5 \mathrm{nM}$ ). The control group was treated with a volume of phosphatebuffered saline (PBS) equal to the volume of the drug solution that was added in the other groups. Dihydroethidium (DHE) $(0.5 \mathrm{~mL}$ at $0.5 \mu \mathrm{M})$ was added to the DMEM in each well and incubated for $20 \mathrm{~min}$, then the cells were washed twice with PBS and visualized using an inverted fluorescence microscope (535-nm excitation, 635-nm emission). In order to quantify the intracellular ROS contents, the intensity of fluorescence was evaluated using image pro plus software (Media Cybernetics, Carlsbad, CA).

\section{RNA Extraction and qRT-PCR}

The PC-3 prostate cancer cells were seeded in 12-well plates at $2 \times 10^{5}$ cells/well and cultured for $24 \mathrm{~h}\left(37^{\circ} \mathrm{C}, 5 \% \mathrm{CO}_{2}\right)$. After $24 \mathrm{~h}$, the culture medium was changed to Roswell Park Memorial Institute (RPMI)-1640 (free of fetal serum). Que, PTX and Que+PTX $(20 \mu \mathrm{M}+5 \mathrm{~nm})$ were added to the wells. After incubating for $48 \mathrm{~h}$, the total cellular RNA was extracted using TRIzol. RT-PCR and qPCR were performed using standard procedures. Table 1 shows the primers used in this study. The data were analyzed using the $\Delta \Delta \mathrm{Ct}$ method, with the $\mathrm{Ct}$ value of GAPDH as the standard, using the equation: $\Delta \mathrm{Ct}=\mathrm{Ct}$ value of targeted gene $\mathrm{Ct}$ value of GAPDH. The targeted gene expression was reported as $2^{-\Delta \Delta \mathrm{Ct}}$.

\section{Western Blotting}

The PC-3 cells were plated in 6-well plates at a concentration of $5 \times 10^{5}$ cells/well, and incubated for $24 \mathrm{hrs}$. The cells were lysed with radio-immunoprecipitation assay (RIPA) lysis buffer supplemented with the protease inhibitor, phenylmethylsulfonyl fluoride $(1 \mathrm{mM})$. A bicinchoninic acid protein assay (Thermo Fisher Scientific, Waltham, MA, USA) was used to determine the protein concentration. The proteins were separated by sodium dodecyl sulfatepolyacrylamide gel electrophoresis and transferred to nitrocellulose membranes (Millipore, Massachusetts, USA). The membrane was incubated with one of the primary antibodies, GRP78 (1:1000), CHOP (1:2000), hnRNPA1 (1:1000), cleaved caspase-3 (1:1000), or GAPDH (1:2000), and incubated with HRP-conjugated secondary goat anti-rabbit or anti-mouse antibody at $37^{\circ} \mathrm{C}$ for $1 \mathrm{~h}$. The images were captured using a Bio-Rad imaging system (Shanghai, China).

\section{Cell Cycle and Apoptosis Evaluation}

The PC-3 cells were plated separately into 6-well culture plates at $5 \times 10^{5}$ cells/well and incubated for $24 \mathrm{~h}$ at $37^{\circ} \mathrm{C}$. The cells were further incubated for $24 \mathrm{~h}$ with Que, PTX, or Que+PTX at equivalent concentrations of Que $(20 \mu \mathrm{M})$ or PTX ( $5 \mathrm{nM}$ ) for $24 \mathrm{~h}$. Subsequently, the cells were harvested at predetermined time points and fixed in $1 \mathrm{~mL}$ cold $70 \%$ ethanol for $24 \mathrm{~h}$. Next, the cells were centrifuged, washed, and incubated with $0.1 \%$ RNase A for $1 \mathrm{~h}$ at $37^{\circ} \mathrm{C}$. Finally, the cells were stained with propidium iodide (PI) for $30 \mathrm{~min}$ at $4^{\circ} \mathrm{C}$ and analyzed using flow cytometry (Becton Dickinson, New York, USA). The untreated cells were used as the negative control.

Cell apoptosis was assessed using Annexin V-PI (Byotime Co., Ltd, Haimen, China). Briefly, the PC-3 cells were seeded onto a 6-well plate at a concentration of $4 \times 10^{5}$ cells/well and incubated for $24 \mathrm{~h}$. The PC-3 cells were then incubated with Que, PTX, or Que+PTX at an equivalent concentration of Que $(20 \mu \mathrm{M})$ or PTX $(5 \mathrm{nM})$ for $24 \mathrm{~h}$. Next, cells were collected and centrifuged (1500 rpm for $5 \mathrm{~min}$ ), resuspended in PBS, and incubated with $195 \mu \mathrm{L}$ of binding solution. The cells were then incubated with $5 \mu \mathrm{L}$ of Annexin V-FITC and $10 \mu \mathrm{L}$ of PI solution. After incubation for $15 \mathrm{~min}$ at room temperature, the cells were analyzed using FACScan flow cytometry (Becton Dickinson, New York, USA). The cells that were not treated with Annexin V-PI were used as controls.

\section{Cell Migration Analysis}

Cell migration assays were performed using Transwell chambers $(6.5 \mathrm{~mm})$ with 8 - $\mu \mathrm{m}$ pores in 24 -well plates.

Table I Primers Used in This Study

\begin{tabular}{|l|l|l|l|}
\hline Name & Forward (5' to $\mathbf{3}^{\prime}$ ) & Reverse (5' to 3') & Expected Size (bp) \\
\hline GRP78 & GGAACCATCCCGTGGCATAA & CTTGGTAGGCACCACTGTGT & 193 \\
CHOP & CCACCACACCTGAAAGCAGAA & GGTGCCCCCAATTTCATCT & 148 \\
Shh & GTGGCCGAGAAGACCCTA & CAAAGCGTTCAACTTGTCCTTA & 177 \\
hnRNPAI & AACGCTCACGGACTGTGTGGTAAT & GTGGCCTTGCATTCATAGCTGCAT & 116 \\
GAPDH & GTCAGTGGTGGACCTGACCT & TGCTGTAGCCAAATTCGTTG & 245 \\
\hline
\end{tabular}


The PC-3 cells were treated with Que, PTX, or Que+PTX. The treated cells were added to the upper chamber in serum-free medium, while the medium with $10 \%$ FBS was added to the lower chamber, which was used as a chemoattractant. The plates were incubated for $20 \mathrm{~min}$, fixed with $0.1 \%$ glutaraldehyde for $20 \mathrm{~min}$ and stained with crystal violet $(0.2 \%)$. Images were acquired using an inverted microscope equipped with an IX-51 fluorescence microscope from Olympus Optical Company, Ltd. (Tokyo, Japan), and the total number of cells was counted.

\section{Therapeutic Effects}

The PC-3 prostate cancer-bearing cells were established in nude male $\mathrm{BALB} / \mathrm{c}$ mice by subcutaneously inoculating a PC-3 cell suspension $\left(2 \times 10^{6}\right.$ cells $\left./ 100 \mu \mathrm{L}\right)$ into the mice's armpits. The animal experiments were conducted once the tumor volume reached approximately $50 \mathrm{~mm}^{3}$. The PC-3 cancer-bearing mice were then randomly divided into four groups of six mice each. PBS, Que, PTX, or Que+PTX were injected into the cancer-bearing mice through the tail vein. The mice were treated every other day for 21 days. During the prostate cancer therapy, the animals were weighed every other day, and the surviving mice were monitored throughout the experiment. The tumor size was measured using a Vernier caliper, and the tumor volume $(\mathrm{V})$ was calculated as $\mathrm{V}=\mathrm{d} 2 * \mathrm{D} / 2$, where $\mathrm{d}$ and $\mathrm{D}$ corresponded to the shortest and longest diameters of the tumor in $\mathrm{mm}$, respectively. After the treatment period, the mice were sacrificed; their heart, liver, spleen, lung, and kidneys were obtained, and the organ morphologies were observed.

\section{Immunohistochemistry}

The tumors were resected from the mice, processed, and cut into 5- $\mu \mathrm{m}$ sections using a microtome (Leica RM2165, Leica Microsystems, Wetzlar, Germany). The sections were mounted on glass slides and stained with the antibodies for GRP78 (1:100), CHOP (1:100), hnRNPA1(1:100), and cleaved caspase-3 (1:100). The slides were then incubated with peroxidase-conjugated secondary antibody, and 3,3'diaminobenzidine (DAB) reagent was used as the chromogen. All immunostaining was performed using the Ventana Benchmark automated immunostainer (Ventana Medical Systems, Tucson, AZ, USA). Histoscores were applied to interpret the results. Briefly, staining intensity was graded as negative (0), weak (1), moderate (2) or strong (3), the score was multiplied by the percentage of tumor cells within each category. ${ }^{15}$ The final histoscores ranged from 0 to 300 . Two observers, who were blinded to all outcome data, graded the GRP78/BiP, CHOP, hnRNPA1 and cleaved caspase-3 staining. The images were acquired using an inverted microscope equipped with an IX-51 fluorescence microscope from Olympus Optical Company, Ltd. (Tokyo, Japan).

\section{Statistical Analysis}

The relevant data were analyzed using SPSS 13.0 statistical software. The mean \pm standard deviation $(\mathrm{x} \pm \mathrm{s})$ was used to represent the experimental data. A $t$-test was used to compare the means between two independent samples; a oneway analysis of variance (ANOVA) was used to measure the differences between the means of multiple samples. The groups were compared using the Student-Newman-Keuls (SNK) method, and $P<0.05$ was considered to be statistically significant.

\section{Results}

\section{Toxicity Toward PC-3 Cells}

We performed an MTT assay to evaluate the effect of Que on the sensitivity of prostate cancer cells to PTX. The inhibitory concentration (IC50) of Que to the PC-3 cells was $50.9 \mu \mathrm{M}$ (Figure 1A), and the IC50 of PTX to the PC3 cells was $22.2 \mathrm{nM}$ (Figure 1B). When the cancer cells were treated with Que $(15 \mu \mathrm{M})$, PTX $(12.5 \mathrm{nM})$, or Que+PTX (15 $\mu \mathrm{M}+12.5 \mathrm{nM}$ ) (Figure $1 \mathrm{C}$ ), the growth inhibitory rates were $17.01 \pm 1.09 \%, 24 \pm 2.33 \%$, and $73 \pm 3.90 \%$, respectively, and the combination index (CI) was 0.55 , which suggested a synergistic effect (Figure 1D). Thus, Que increased the cell killing effects of PTX, and $12.5 \mathrm{nM}$ of PTX, when combined with Que, killed nearly half the cancer cells.

\section{Anticancer Mechanism of Combined Low-Dose Que and PTX}

Because Que exerted anticancer effects at a low dose, we used $20 \mu \mathrm{M}$ Que and $5 \mathrm{nM}$ PTX to evaluate cancer cell apoptosis. We used Annexin V-PI double staining to evaluate whether apoptosis was attributed to cell death. Cells treated with Que (Figure 2B), PTX (Figure 2C), and Que+PTX (Figure 2D) yielded total apoptotic rates of $7.4 \pm 3.09 \%, 11.8 \pm 4.33 \%$, and $45 \pm 3.90 \%$, respectively, while the apoptotic rate of the control group was $1.5 \pm 0.4 \%$ (Figure 2A). Que+PTX had the highest total apoptotic rate at $45 \%$, which was higher than that in the single Que and PTX groups. The early apoptosis rate and the late apoptosis rate of the combinational treatment group were also much more, when compared with single treatment (Figure 2E). These outcomes indicated that Que 

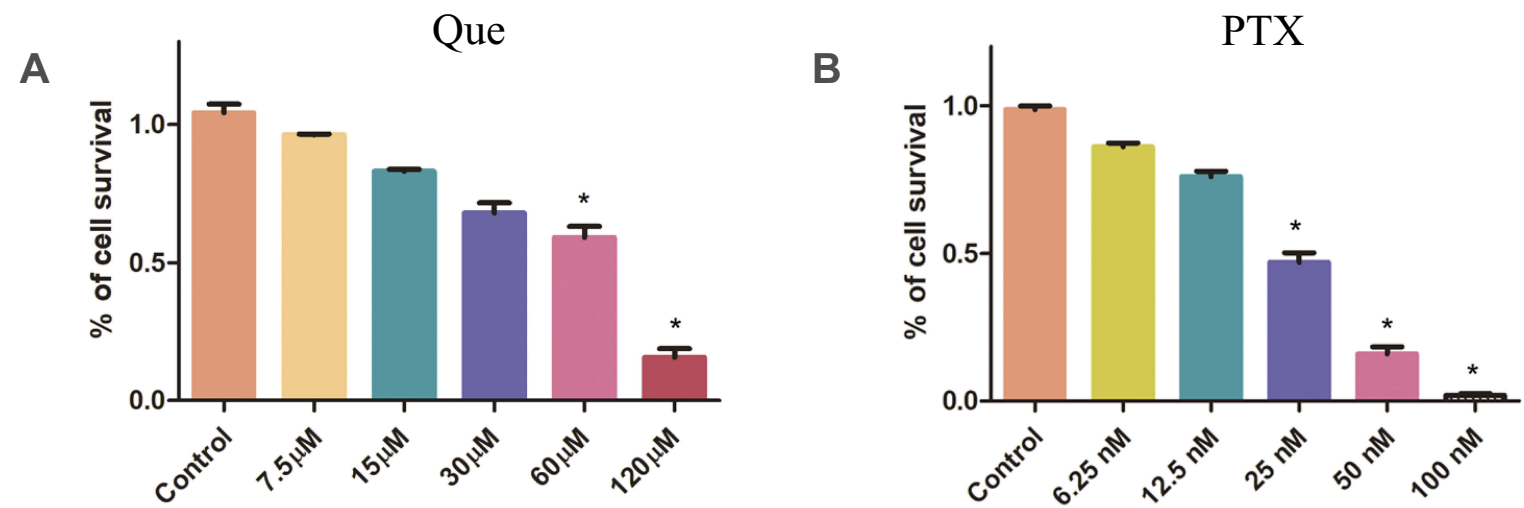

C

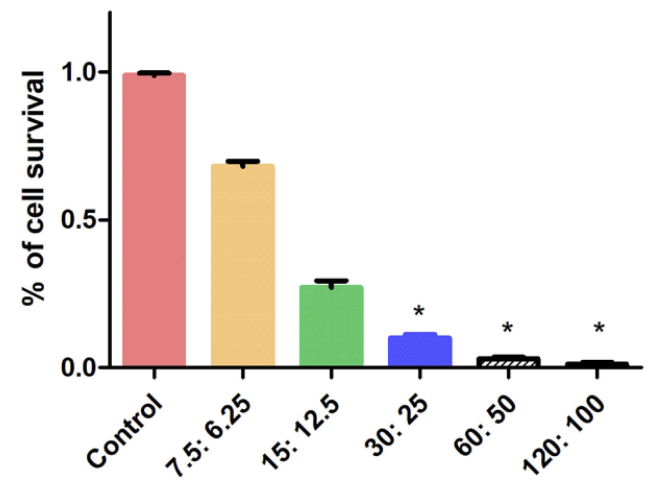

D

Que: PTX

Figure I Quercetin or (plus) paclitaxel inhibits PC-3 cell viability. (A) Quercetin cytotoxicity towards PC-3 cells; (B) Paclitaxel cytotoxicity toward PC-3 cells; and (C) Cytotoxicity of quercetin in combination with paclitaxel toward PC-3 cells; (D) The Chou and Talalay method was used for the analysis of the Combination Index (Cl) of the quercetin and paclitaxel combination. Cell viability was detected in PC-3 cells after treatment of varying concentrations of drug at 24 by MTT methods. $n=3, * P<0.05$.

sensitized PTX to induce cancer cell early and late apoptosis. In line with these results, cleaved caspase-3 protein was also noticeably detected in this combination treatment group (Figure 5D), which is a marker of cellular apoptosis.

\section{Que Induced Cell Cycle Arrest in the G2/ M Phase}

PI staining was used to evaluate the cell cycle of PC-3 cells treated with Que, PTX, or Que+PTX. The cell cycle was analyzed to evaluate whether Que inhibited cell cycle progression. The cells were exposed to $20 \mu \mathrm{M}$ Que and 5 nM PTX either singly or combined. The G2/M phase in the combined group (Figure 3D) was $20.58 \%$, while it was $8.36 \%$ in the control group (Figure 3A), 14.2\% in the Que group (Figure 3B), and $18.09 \%$ in the PTX group (Figure 3C). The combined group possessed the most potent ability to induce cell cycle arrest at the G2/M phase compared with the single PTX or single Que groups. These results indicated that Que increased PTX's effect on cell cycle arrest (Figure 3E). These results also explain the combined group's synergistic effect on PC-3 cancer cell death.

\section{Que Induced Reactive Oxygen Species Production}

DHE is a commonly used fluorescent probe for detecting intracellular ROS. DHE can passively diffuse into cells, and because it is highly reactive, it can react with cytosolic superoxide. DHE is then converted to red fluorescent 2-hydroxyethidium, which is visible if the cell contains ROS. We treated the PC-3 cancer cells with Que, PTX, or Que+PTX (Figure 4A). The results showed that Que induced much more ROS generation compared with the control group, and that the ROS contents in the Que group was much more than that in the PTX group. The PC3 cells treated with PTX failed to induce ROS as compared to control, and the ROS content was not induced significantly as compared with the control group. This indicated 
A

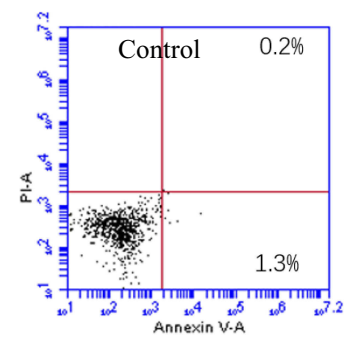

C

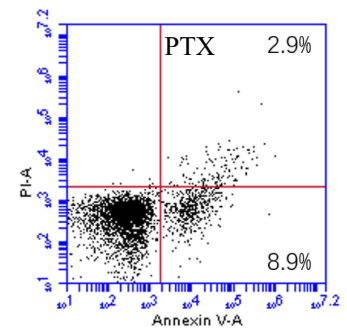

B

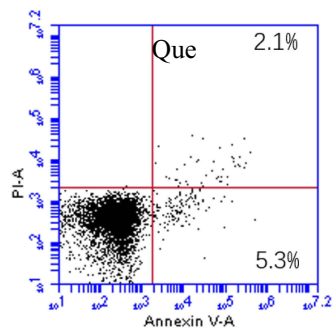

D

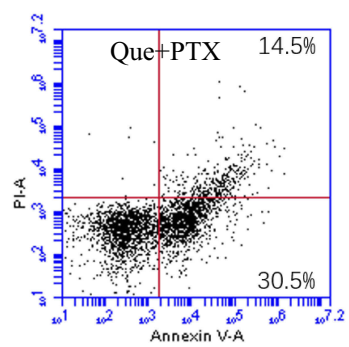

E

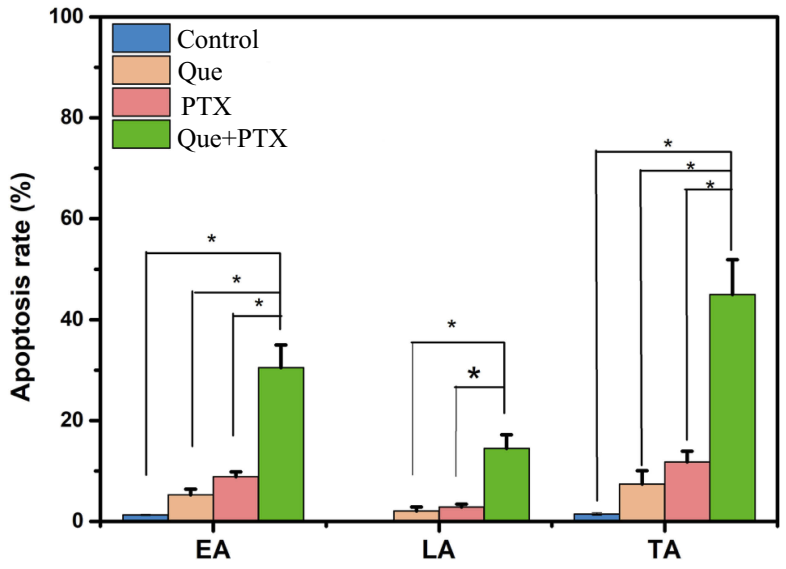

Figure 2 In vitro apoptosis rate of PC-3 cells determined by flow cytometry analysis using Annexin V-FITC and PI double-staining after incubation with PBS (A), quercetin (B), paclitaxel (C), combination quercetin and paclitaxel (D) for 24 h. Early apoptosis (EA), late apoptosis (LA), and total apoptosis (TA) were the most in the combination treatment group (E). $n=3, * P<0.05$.

A

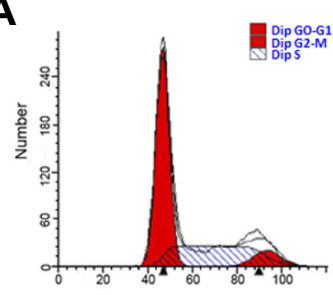

C

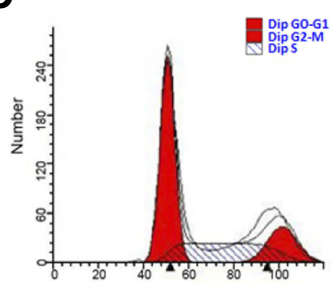

B

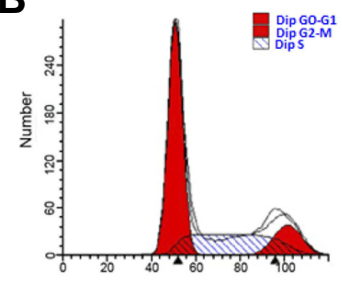

D

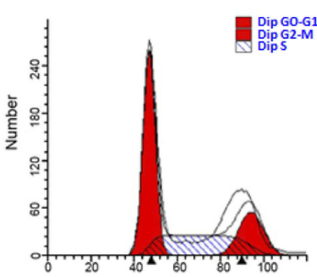

E

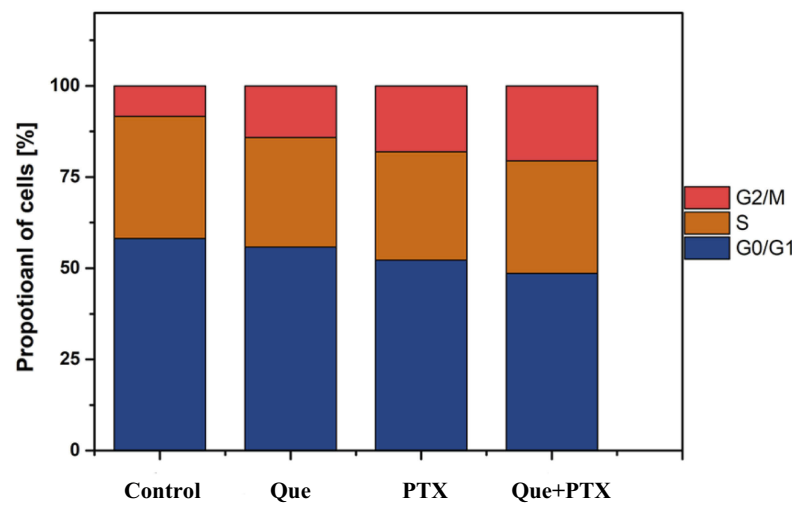

Figure 3 Cell cycle analysis of PC-3 cells was detected using flow cytometric analysis with PI single-staining after incubation with PBS (A), quercetin (B), paclitaxel (C), combination quercetin and paclitaxel (D) for $24 \mathrm{~h}$. The combination treatment group could effectively induce cell cycle arrest at G2/M phase compared with the other group (E).

that the high level ROS in the combination treatment group may be mainly attributed to Que. However, the combined group presented the highest ROS content, which was much more than that of the Que group (Figure 4B). ROS production induces cell death by damaging the DNA and causing a loss of cell plasma integrity, thus inducing apoptosis.

\section{Relative Gene Expression Analysis}

First, we evaluated the changes in GRP78, CHOP, and hnRNPA1 gene expressions after treating the PC-3 cells using different formulations of Que and/or PTX. The combined group effectively decreased hnRNPA1 gene expressions and increased the GRP78 and CHOP gene expressions, which are related to ER stress and ROS production (Figure 5). ER 
A

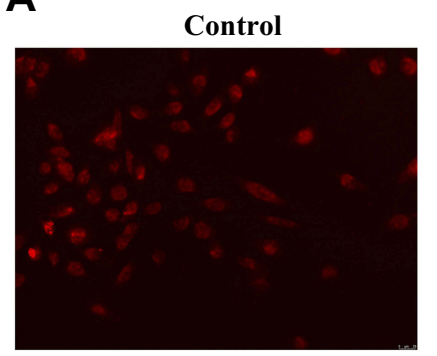

PTX

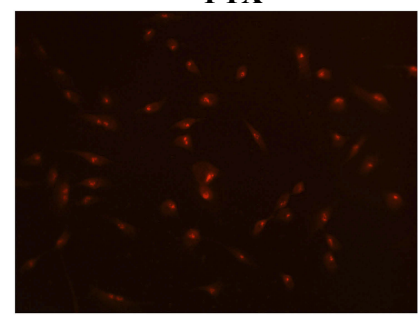

Que

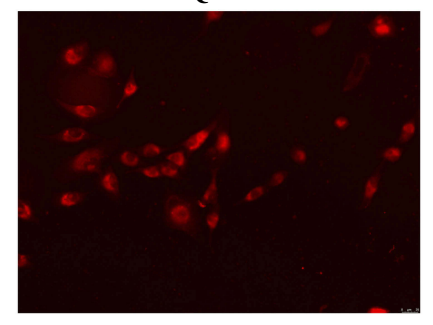

Que+PTX

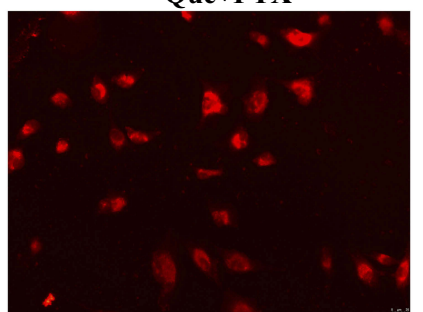

B

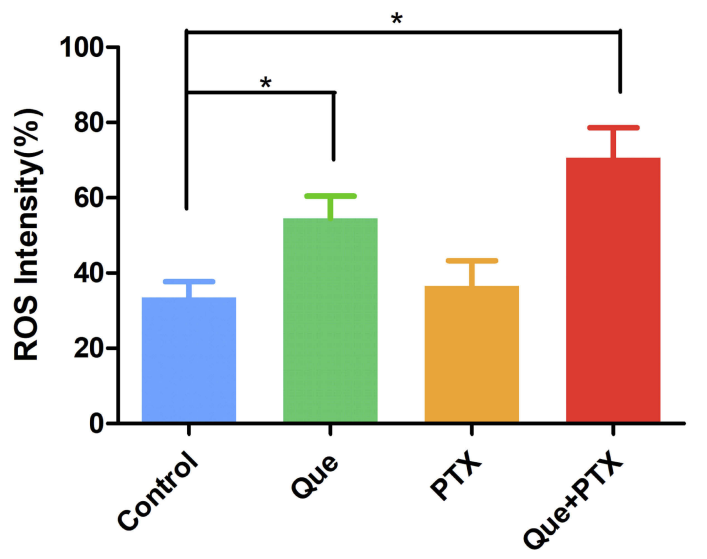

Figure 4 Intracellular ROS content was detected using dihydroethidium (DHE) staining by fluorescence microscopy after the cells were incubated with PBS, quercetin, paclitaxel, combination quercetin and paclitaxel $(\mathbf{A})$ for $24 \mathrm{~h}$, the combination treatment group induced the most contents of ROS $(\mathbf{B})$. $\mathrm{n}=6$, $* P<0.05$.

A

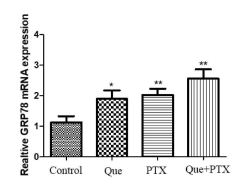

$\mathbf{F}$

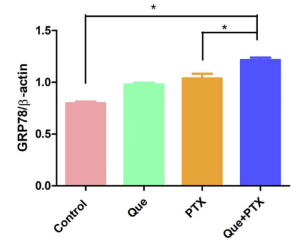

B

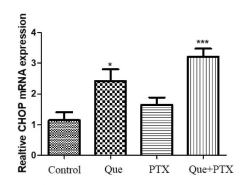

C

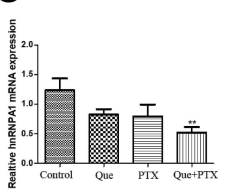

D

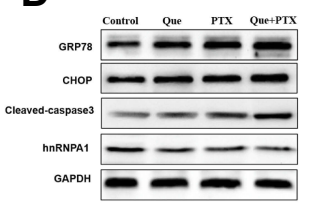

$\mathbf{E}$

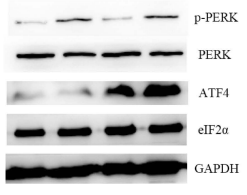

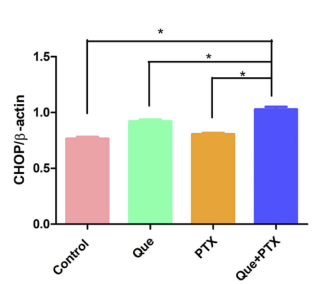
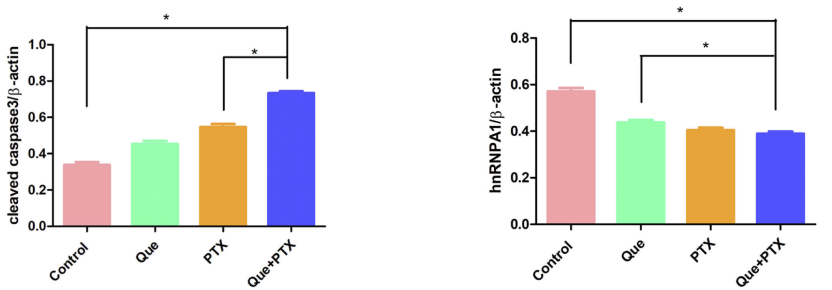

Figure 5 The hnRNPAI, Chop, and GRP78 expressions in gene and protein levels were evaluated by real-time PCR (A-C) or Western blotting (D), after the PC-3 cells were treated with PBS, quercetin, paclitaxel, and combination of quercetin and paclitaxel for $24 \mathrm{~h}$. The ER stress related PERK-elF2 $\alpha$-ATF4 pathway was also evaluated by Western blotting (E); the protein changes in GRP78, chop, cleaved caspase 3 and hnRNPAI were quantified using Image J software $(\mathbf{F})$. $n=3, * P<0.05, * * P<0.0$ I, $* * * P<0.005$.

stress related PERK-eIF2 $\alpha$-ATF4 pathway was also evaluated, and the protein p-PERK and ATF4 increased after cells being treated with quercetin. Downregulating hnRNPA1 made the cells prone to being killed by PTX. After hnRNPA1 was downregulated by Que, its migration abilities were assessed using transwell assay, and the combination treatment effectively inhibited prostate cancer cell migration compared with single treatment (Figure 6).

\section{In vivo Tumor Growth Inhibition}

The PC-3 tumor xenografts were established to study the in vivo therapeutic efficiency of using Que+PTX. Nude mice bearing PC-3 tumor xenografts were subjected to $50 \mathrm{mg} / \mathrm{kg}$
Que and $5 \mathrm{mg} / \mathrm{kg}$ PTX every other day. Figure 7A and B shows that tumor growth was minimally inhibited in both the Que and PTX groups compared with the control group, which most significantly inhibited tumor growth. This indicated that Que synergized PTX's in vivo antitumor effect. Notably, mice in the single paclitaxel group showed decreases in body weight during the 21-day therapeutic period, while the combination treatment group did not show this side effect (Figure 7C), which may be due to the side effect of the PTX.

\section{In vivo Protein Expression}

A histological analysis showed necrosis in the Que + PTX group. Immunohistochemistry showed that the 


\section{A}

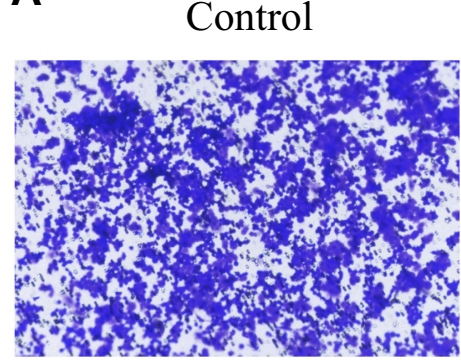

PTX

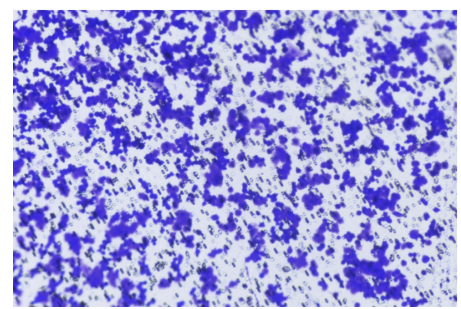

Que

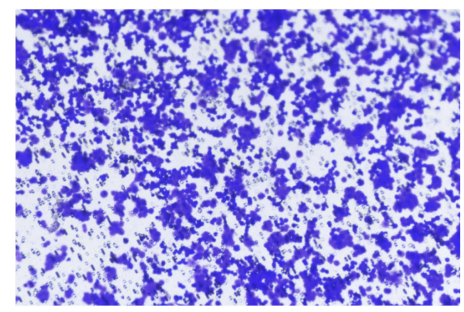

Que+PTX

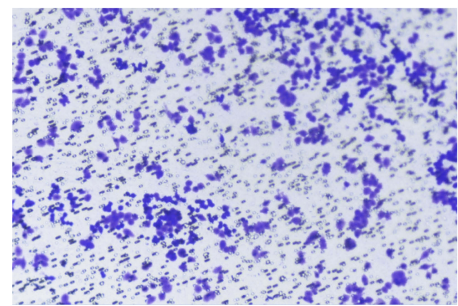

B

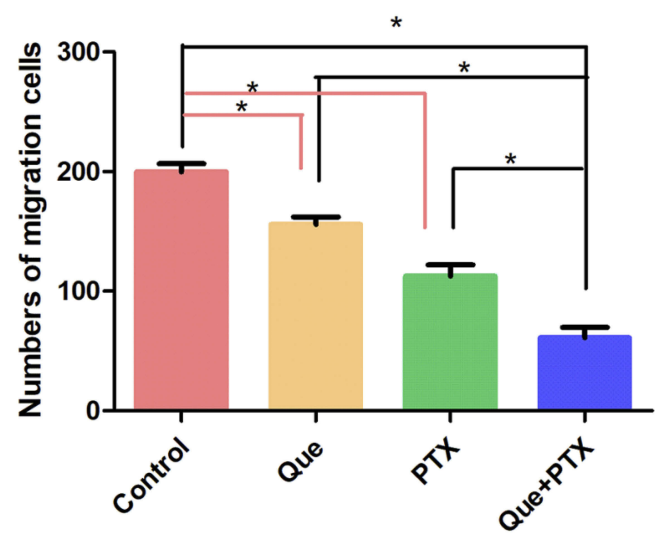

Figure 6 The transwell method was used to evaluate the PC-3 cell migration after the PC-3 cells were incubated with PBS, quercetin, paclitaxel, combination quercetin and paclitaxel (A). The combination treatment group could effectively inhibit cell migration compared with the other group (B). $n=3, * P<0.05$.

cleaved-caspase-3 expression increased in the Que+PTX group compared with the uncombined Que and PTX groups, indicating that the combination treatment can effectively induce cancer cells to undergo apoptosis. CHOP and GRP78 expressions were also noticeably increased when compared with the single treatment, indicating that the combined treatment effectively induced ER stress and ROS production to induce cancer cell death. Consistent with the in vitro Western blot results, hnRNPA1 was slightly decreased in the combined treatment group, which may be due to the Que (Figure 8).

\section{Discussion}

Traditional chemotherapy presents many problems such as adverse effects, poor tolerance among patients, drug resistance, and lack of a targeted effect. PTX is a model drug widely used in clinical oncological treatment; however, it induces adverse effects, including bone marrow inhibition and vomiting. One important problem with PTX is that when used for a long time, patients can develop drug resistance, which decreases the drug's antitumor effects. Alternative therapies are attracting attention for their advantages such as greater efficiency and less toxicity. Accordingly, Que has been indicated as a prominent target in investigations of alternative therapies against cancer. ${ }^{16,17}$

In this study, Que and PTX were combined to treat prostate cancer and investigate the anticancer effect of combined therapy. The PC-3 cells are model cells of castration-resistant prostate cancer, which is a phenomenon that often occurs with prostate cancer after castration treatment, and often leads to the patient's death. ${ }^{18}$ We found that Que synergized PTX to kill the PC-3 cancer cells, and that PC-3 cell proliferation was efficiently inhibited in the combined therapy group. The $5 \mathrm{nM}$ concentration of PTX showed much fewer cell-killing effects toward PC-3 cells, but when combined with Que, the inhibitory rate was $51.44 \%$. This finding was consistent with the outcome in previous studies showing that Que increased the sensitivity of ovarian cancer cells to cisplatin and PTX. ${ }^{19}$

Proliferation assays of PC-3 cells treated with PTX alone or Que+PTX indicated that additive or synergistic effects existed between PTX and Que. In addition, single or combined treatments with Que and PTX showed different effects on PC-3 cell biological behaviors such as proliferation, apoptosis, cell cycle, and migration. Furthermore, the combination treatment induced ER stress and then ROS production, which damaged the DNA integrity. The tumor xenograft models showed that the combined treatment effectively inhibited tumor growth better than the single treatment, and cleaved caspase-3, CHOP, and GRP78 were upregulated, while the hnRNPA1 protein was downregulated.

A DHE fluorescence probe was used to detect the intracellular ROS contents, and Que alone induced ROS production, while the combination treatment increased the intracellular ROS contents. Intracellular ROS may induce 
A

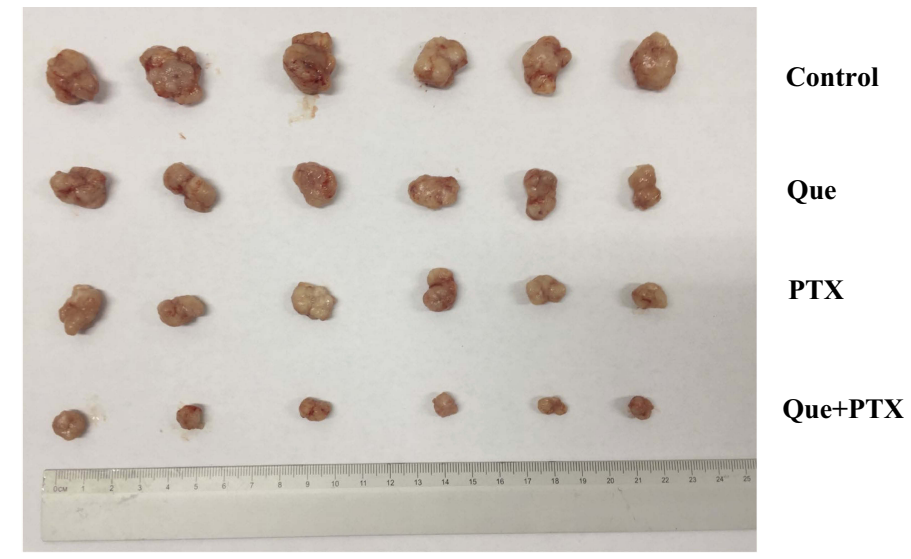

B

C
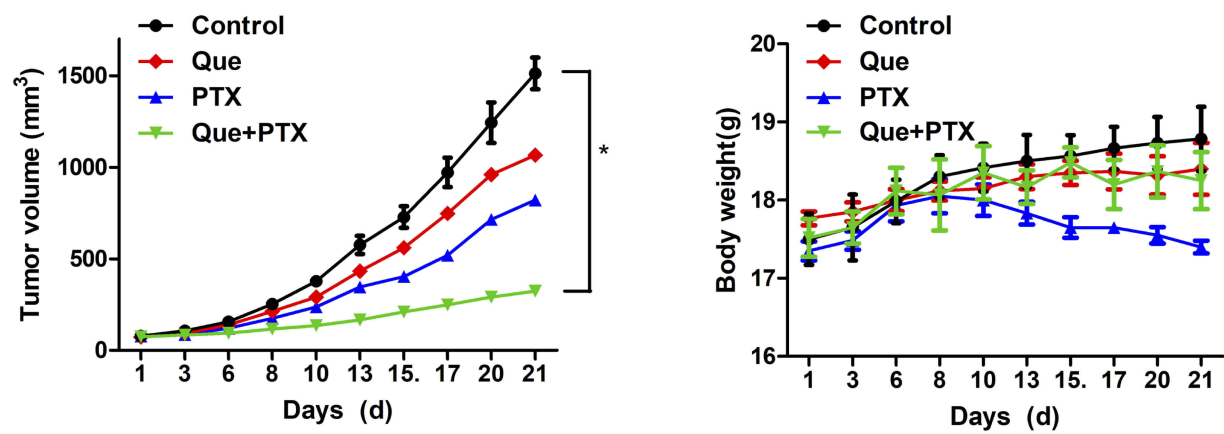

Figure 7 In vivo anti-cancer activity of quercetin or paclitaxel alone or combination against the PC-3 cancer-bearing mice, the combination treatment could effectively inhibit cancer growth. Tumor gross picture (A); Cancer growth curves (B); and body weight change curves (C). $n=6, * P<0.05$.

DNA damage and chromosome instability and activate apoptotic pathways to induce cancer cell death. ${ }^{20}$ Several studies have reported that Que has an antioxidative effect that can quench ROS, reactive nitrogen species, and other radicals. $^{21,22}$ Li et al showed that low concentration of Que decreased the cytotoxic effect of cisplatin by reducing intracellular ROS in ovarian cancer. ${ }^{23}$ While one study showed that quercetin could antagonize the G2/M arrest induced by taxol and nocodazole, the combination treatment of taxol and Que could synergistically inhibit the colony formation of HCT116 colon cancer cell. ${ }^{24}$ This phenomenon may be related to different cancer cell types and different Que concentrations.

Interestingly, the two proteins GRP78 and CHOP, which are related to ER stress, were upregulated, which coincided with the increased ROS. Upregulating the mRNA and protein levels of CHOP and GRP78 showed that Que effectively induced ER stress, and that ER stress may contribute to cancer cell apoptosis and death. One study showed that Que induced human cervical carcinoma
Hela cell apoptosis via the ER-stress pathway. ${ }^{23}$ Another study showed that quercetin enhanced the radiosensitivity of human ovarian cancer cells via the p53-dependent ER stress pathway. ${ }^{24}$

PTX induces cell cycle arrest at the G2/M phase, leading to cancer cell death. Que regulates the cell cycle by modulating several cell cycle-associated proteins, such as p21, cyclin B, p27, and cyclin-dependent kinase. ${ }^{25}$ Another study showed that Que induced esophageal squamous cell cycle arrest at the G2/M phase by upregulating p21waf1 and downregulating cyclin B1. ${ }^{26}$ In lung cancer cells, Que induces cell cycle arrest at the G2/M phase by upregulating cyclin B and Cdc25c-ser-216-p. ${ }^{27}$ Que not only affects the G2/M phase, but several studies have shown that it induces cell cycle arrest at the G1 phase in HepG2 hepatomas. These phenomena may be related to cell types. ${ }^{28}$ In this study, we found that quercetin induced cell cycle arrest at the G2/M phase and that the combination treatment possessed a synergistic cell-cycle-blocking effect. 


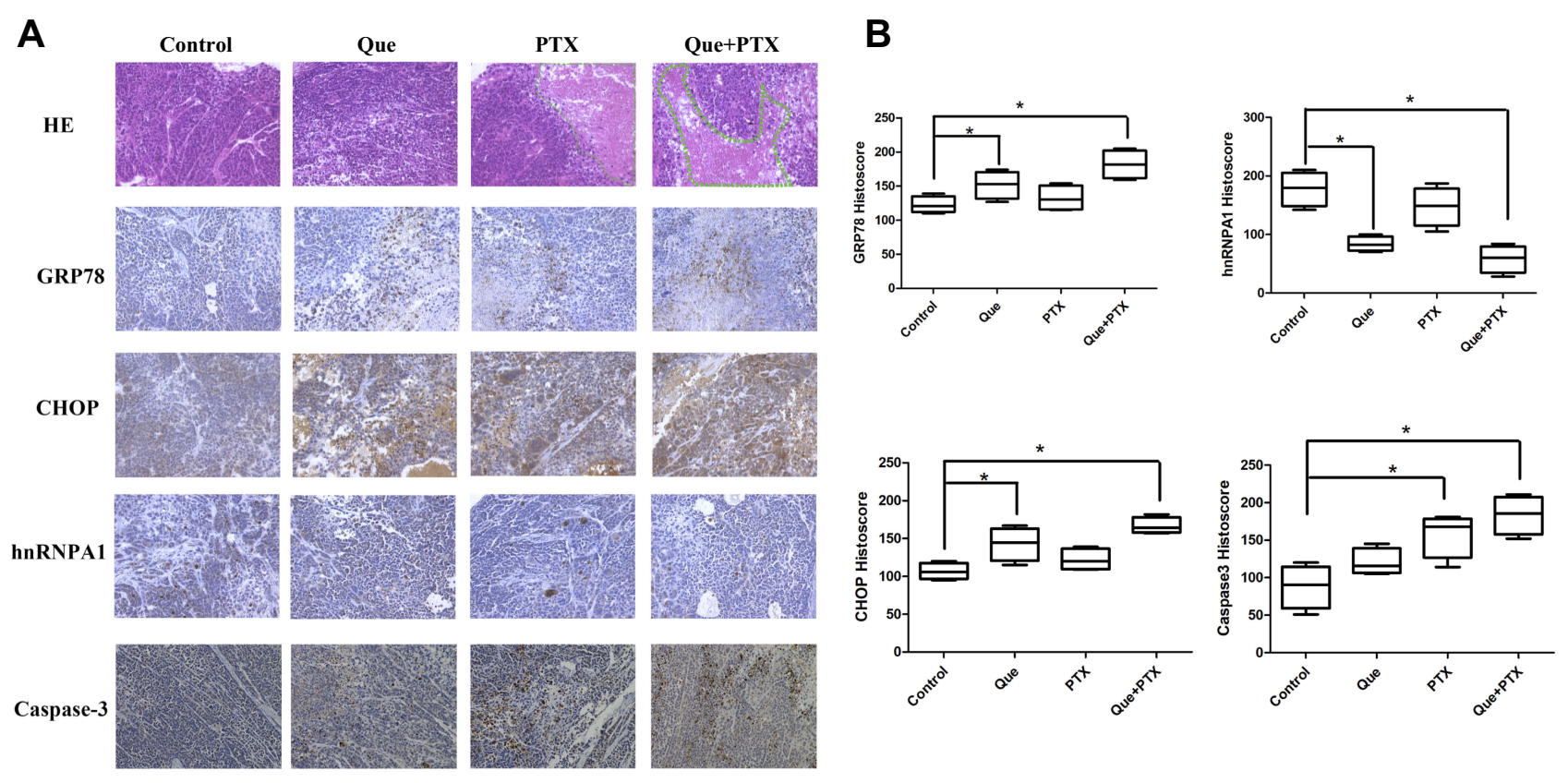

Figure 8 In vivo expressions of protein GRP78, CHOP, hnRNPAI, CHOP, and cleaved caspase 3 expression in PC-3 xenografts after being treated with PBS, quercetin, paclitaxel, and combination of quercetin and paclitaxel (A). The protein expression level was evaluated using immunohistochemistry, the combination treatment group could effectively increase the expression of GRP78, CHOP and cleaved caspase 3, while downregulate the expression of hnRNPAI (B). n=6, $* P<0.05$.

The hnRNPA1 protein was a molecule target of Que in PC-3 cells, ${ }^{29}$ and overexpression of hnRNPA1 was related to cancer cell epithelial-to-mesenchymal transition (EMT), which was considered to be a mechanism of cancer cell migration. ${ }^{30,31}$ Therefore, hnRNPA1 may be related to cell migration and metastasis, quercetin could decrease the hnRNPA1 expression to inhibit EMT of cancer cell. In this study, transwell assays revealed that Que combined with PTX effectively inhibited cell migration. We evaluated the hnRNPA1 mRNA and protein levels of PC-3 cells and found that hnRNPA1 was downregulated in the in vitro cell experiment, which was consistent with previous studies. We also evaluated hnRNPA1 protein expression in the tumor section of the in vivo prostate cancer xenograft using immunohistochemistry and found that hnRNPA1 was downregulated in the in vivo tumor model. Que may interact with hnRNPA1 to inhibit prostate cancer cell migration. Moreover, hnRNPA1 was previously reported to be involved in cancer resistance, and we found that Que inhibited the hnRNPA1; therefore, Que may also synergized PTX's cancer cell killing ability by inhibiting hnRNPA1.

In conclusion, this study provided novel evidence for combinational therapies using Que and PTX to treat prostate cancer. We found that Que synergized PTX to inhibit prostate cancer cell proliferation, induce cell cycle arrest at the $\mathrm{G} 2 / \mathrm{M}$ phase, and induce cell apoptosis. In addition, Que effectively induced intracellular ROS production and
ER stress, and the down-regulation of hnRNPA1 may underlie cell migration inhibition. This study may provide insight into prostate cancer clinical treatment and may provide evidence for using Que as a promising therapeutic agent for treating patients with prostate cancer.

\section{Acknowledgments}

This research was supported, in part, by the Natural Science Foundation of Shandong Province (No. ZR2017QH005) and the National Natural Science Foundation of China (Nos. 81803097 and 81602727). This work was also supported in part by the Program of Natural Science Foundation of the Anhui Higher Education Institutions (No. KJ2019A0313).

\section{Disclosure}

The authors declare that they have no potential conflicts of interest to disclose in this work.

\section{References}

1. Bray F, Ferlay J, Soerjomataram I, Siegel RL, Torre LA, Jemal A. Global cancer statistics 2018: GLOBOCAN estimates of incidence and mortality worldwide for 36 cancers in 185 countries. CA Cancer J Clin. 2018;68(6):394-424. doi:10.3322/caac.v68.6

2. Chen W, Zheng R, Baade PD, et al. Cancer statistics in China, 2015. CA Cancer J Clin. 2016;66(2):115-132. doi:10.3322/caac.21338

3. Valenca LB, Sweeney CJ, Pomerantz MM. Sequencing current therapies in the treatment of metastatic prostate cancer. Cancer Treat Rev. 2015;41(4):332-340. doi:10.1016/j.ctrv.2015.02.010 
4. Galletti G, Leach BI, Lam L, Tagawa ST. Mechanisms of resistance to systemic therapy in metastatic castration-resistant prostate cancer Cancer Treat Rev. 2017;57:16-27. doi:10.1016/j.ctrv.2017.04.008

5. Perez EA. Differentiating tubulin-inhibiting agents based on mechanisms of action, clinical activity, and resistance. Mol Cancer Ther. 2009;8(8):2086-2095. doi:10.1158/1535-7163.MCT-09-0366

6. Lanzi C, Cassinelli G, Cuccuru G, et al. Cell cycle checkpoint efficiency and cellular response to paclitaxel in prostate cancer cells. Prostate. 2001;48(4):254-264. doi:10.1002/(ISSN)1097-0045

7. Sobue S, Mizutani N, Aoyama Y, et al. Mechanism of paclitaxel resistance in a human prostate cancer cell line, PC3-PR, and its sensitization by cabazitaxel. Biochem Biophys Res Commun. 2016;479(4):808-813. doi:10.1016/j.bbrc.2016.09.128

8. Kars MD, Işeri OD, Gunduz U, Molnar J. Reversal of multidrug resistance by synthetic and natural compounds in drug-resistant MCF-7 cell lines. Chemotherapy. 2008;54(3):194-200. doi:10.1159/000140462

9. Fojo T, Bates S. Strategies for reversing drug resistance. Oncogene. 2003;22(47):7512-7523. doi:10.1038/sj.onc.1206951

10. Iheagwam FN, Ogunlana OO, Ogunlana OE, Isewon I, Oyelade J. Potential anti-cancer flavonoids isolated from caesalpinia bonduc young twigs and leaves: molecular docking and in silico studies. Bioinform Biol Insights. 2019;13:1177932218821371. doi:10.1177/1177932218821371

11. Jana N, Břetislav G, Pavel S, Pavla U. Potential of the flavonoid quercetin to prevent and treat cancer - current status of research. Klin Onkol. 2018;31(3):184-190. doi:10.14735/amko2018184

12. Jeon JS, Kwon S, Ban K, et al. Regulation of the intracellular ROS level is critical for the antiproliferative effect of quercetin in the hepatocellular carcinoma cell line HepG2. Nutr Cancer. 2019;19:1-9.

13. Gibellini L, Pinti M, Nasi M, et al. Quercetin and cancer chemoprevention. Evid Based Complement Alternat Med. 2011;2011:591356. doi:10.1093/ecam/neq053

14. Tummala R, Lou W, Gao AC, Nadiminty N. Quercetin targets hnRNPA1 to overcome enzalutamide resistance in prostate cancer cells. Mol Cancer Ther. 2017;16(12):2770-2779. doi:10.1158/15357163.MCT-17-0030

15. Kirkegaard T, Edwards J, Tovey S, et al. Observer variation in immunohistochemical analysis of protein expression, time for a change? Histopathology. 2006;48(7):787-794. doi:10.1111/ his.2006.48.issue-7

16. Houghton PJ. The role of plants in traditional medicine and current therapy. J Altern Complement Med. 1995;1(2):131-143. doi:10.1089/ acm.1995.1.131

17. Zhao J, Fang Z, Zha Z, et al. Quercetin inhibits cell viability, migration and invasion by regulating miR-16/HOXA10 axis in oral cancer. Eur J Pharmacol. 2019;847:11-18. doi:10.1016/j.ejphar.2019.01.006

18. Monn MF, Tatem AJ, Cheng L. Prevalence and management of prostate cancer among East Asian men: current trends and future perspectives. Urol Oncol. 2016;34(2):58.e1-58.e9. doi:10.1016/j. urolonc.2015.09.003
19. Maciejczyk A, Surowiak P. Quercetin inhibits proliferation and increases sensitivity of ovarian cancer cells to cisplatin and paclitaxel. Ginekol Pol. 2013;84(7):590-595. doi:10.17772/gp/1609

20. Kruk J, Aboul-Enein HY. Reactive oxygen and nitrogen species in carcinogenesis: implications of oxidative stress on the progression and development of several cancer types. Mini Rev Med Chem. 2017;17(11):904-919. doi:10.2174/1389557517666170228115324

21. Dajas F. Life or death: neuroprotective and anticancer effects of quercetin. J Ethnopharmacol. 2012;143(2):383-396. doi:10.1016/j. jep.2012.07.005

22. Cai X, Fang Z, Dou J, Yu A, Zhai G. Bioavailability of quercetin: problems and promises. Curr Med Chem. 2013;20(20):2572-2582. doi:10.2174/09298673113209990120

23. Li N, Sun C, Zhou B, et al. Low concentration of quercetin antagonizes the cytotoxic effects of anti-neoplastic drugs in ovarian cancer. PLoS One. 2014;9(7):e100314. doi:10.1371/journal.pone.0100314

24. Samuel T, Fadlalla K, Turner T, Yehualaeshet TE. The flavonoid quercetin transiently inhibits the activity of taxol and nocodazole through interference with the cell cycle. Nutr Cancer. 2010;62 (8):1025-1035. doi:10.1080/01635581.2010.492087

25. Jeong JH, An JY, Kwon YT, Rhee JG, Lee YJ. Effects of low dose quercetin: cancer cell-specific inhibition of cell cycle progression. $J$ Cell Biochem. 2009;106(1):73-82. doi:10.1002/jcb.21977

26. Zhang Q, Zhao XH, Wang ZJ. Cytotoxicity of flavones and flavonols to a human esophageal squamous cell carcinoma cell line (KYSE-510) by induction of G2/M arrest and apoptosis. Toxicol in Vitro. 2009;23(5):797-807. doi:10.1016/j.tiv.2009.04.007

27. Yang JH, Hsia TC, Kuo HM, et al. Inhibition of lung cancer cell growth by quercetin glucuronides via G2/M arrest and induction of apoptosis. Drug Metab Dispos. 2006;34(2):296-304. doi:10.1124/ dmd.105.005280

28. Mu C, Jia P, Yan Z, Liu X, Li X, Liu H. Quercetin induces cell cycle G1 arrest through elevating Cdk inhibitors p21 and p27 in human hepatoma cell line (HepG2). Methods Find Exp Clin Pharmacol. 2007;29(3):179-183. doi:10.1358/mf.2007.29.3.1092095

29. Ko CC, Chen YJ, Chen CT, et al. Chemical proteomics identifies heterogeneous nuclear ribonucleoprotein (hnRNP) A1 as the molecular target of quercetin in its anti-cancer effects in PC-3 cells. J Biol Chem. 2014;289(32):22078-22089. doi:10.1074/jbc.M114.553248

30. Chen Y, Liu J, Wang W, et al. High expression of hnRNPA1 promotes cell invasion by inducing EMT in gastric cancer. Oncol Rep. 2018;39(4):1693-1701. doi:10.3892/or.2018.6273

31. Tang SN, Singh C, Nall D, Meeker D, Shankar S, Srivastava RK. The dietary bioflavonoid quercetin synergizes with epigallocathechin gallate (EGCG) to inhibit prostate cancer stem cell characteristics, invasion, migration and epithelial-mesenchymal transition. $J \mathrm{Mol}$ Signal. 2010;5:14. doi:10.1186/1750-2187-5-14
OncoTargets and Therapy

\section{Publish your work in this journal}

OncoTargets and Therapy is an international, peer-reviewed, open access journal focusing on the pathological basis of all cancers, potential targets for therapy and treatment protocols employed to improve the management of cancer patients. The journal also focuses on the impact of management programs and new therapeutic

Submit your manuscript here: https://www.dovepress.com/oncotargets-and-therapy-journa agents and protocols on patient perspectives such as quality of life, adherence and satisfaction. The manuscript management system is completely online and includes a very quick and fair peer-review system, which is all easy to use. Visit http://www.dovepress.com/ testimonials.php to read real quotes from published authors. 